\section{Cerebral venous drainage through internal jugular vein}

\author{
Nadiya Y. Mohammed, Giovanni Di \\ Domenico, Mauro Gambaccini \\ Physics and Earth Science Department, \\ University of Ferrara, Italy
}

\begin{abstract}
Internal jugular veins (IJVs) are the largest veins in the neck and are considered the primary cerebral venous drain for the intracranial blood in supine position. Any reduction in their flow could potentially results an increase in cerebral blood volume and intracranial pressure (ICP). The right internal jugular vein communicates with the right atrium via the superior vena cava, in which a functional valve is located at the union of the internal jugular vein and the superior vena cava. The atrium aspiration is the main mechanism governing the rhythmic leaflets movement of internal jugular vein valve synchronizing with the cardiac cycle. Cardiac contractions and intrathoracic pressure changes are reflecting in Doppler spectrum of the internal jugular vein. The evaluation of the jugular venous pulse provides valuable information about cardiac hemodynamics and cardiac filling pressures. The normal jugular venous pulse wave consists of three positive waves, $a, c$, and $v$, and two negative waves, $x$ and $y$. A normal jugular vein gradually reduces its longitudinal diameter, as described in anatomy books; it is possible to segment IJV into three different segments $\mathrm{J}_{3}$ to $\mathrm{J}_{1}$, as it proposed in ultrasound US studies and CT scan. In this review, the morphology and methodology of the cerebral venous drainage through IJV are presented.
\end{abstract}

\section{Introduction}

Internal jugular veins (IJVs) are the largest veins in the neck and are considered the primary cerebral venous drain for the intracranial blood in supine position,,$^{1,2}$ while in upright position, the jugular veins are collapsed, and cerebral venous drain shifts specifically to the non-collapsible vertebral venous plexus. ${ }^{1,3}$

In patients with head injury, cerebral hemorrhage, brain tumors, and hydrocephalus have an impaired hemodynamics, any reduction in IJV flow creates an increase in cerebral blood volume and intracranial pressure ICP., ${ }^{4,5}$
To the contrary, in chronic cerebrospinal venous insufficiency (CCSVI) characterized by stenosis of the IJVs and azygos (AZ) veins, the venous obstructions are compensated by channeling the blood through the collateral circles as alternative pathways or vicarious venous shunts so preventing intracranial hypertension. ${ }^{6-8}$

The evaluation of the jugular venous pulse (JVP) provides valuable information about cardiac hemodynamics and cardiac filling pressure, which is defined as the heart preload at the end of diastole. ${ }^{9,10}$ Further, JVP gives information about the characteristic wave patterns pathologic of cardiac diseases, ${ }^{11}$ and an indirect estimate of the central venous pressure central venous pressure (CVP) which facilitates the diagnosis and prognosis of many heart diseases. ${ }^{12}$

The JVP is defined as the expansion and contraction movement of IJV due to pressure change in right atrium (RA). It reflects the variation in RA pressure loading by cardiac cycle. The RA pressure can modify the pressure gradient which drives the blood from brain to RA. ${ }^{13}$ Hence, it can modulate the IJV blood velocity. ${ }^{14,15}$

IJV is not known only as pulsatile but also it is a distensible vein, for this reason, the IJV adjusts its shape according to the atmospheric pressure and internal venous pressure of this collapsible vein. ${ }^{16-18}$ In ultrasound study (US), it has been shown a strong relation between IJV cross-section area (CSA) and the transmural pressure, which is the difference between internal venous pressure and atmospheric pressure. Therefore, the time diagram of the IJV cross section area obtained in analyzing ultrasound B-mode images reflects on the JVP. ${ }^{19,20}$

Abnormal jugular venous flow was classified into markedly decreased flow velocity; pulsatile turbulent jugular venous flow and reversed flow. The atrium aspiration is the main mechanism governing the rhythmic leaflets movements of internal jugular vein valve (IJVV) being synchronous with the cardiac cycle. ${ }^{21,22}$

The IJVV, located near the junction with the innominate vein, act as a buffer prevent transmission of thoracic pressure and the reflux of jugular venous blood (JVR) into the cerebral circulation. ${ }^{23,24}$ The sustained JVR in abnormal elevated Valsalva maneuver may render the IJV valves incompetent and pose a retrograde transmitted pressure into the central nervous system CNS. ${ }^{25-27}$

IJV outflow disturbance may be secondary to either extra luminal compression caused by enlarged thyroid gland, bony structures and adjacent artery/or intraluminal anomalies including membrane, web,
Correspondence: Giovanni Di Domenico, Department of Physics and Earth Science, Scientific and Technology Pole, University of Ferrara, via Saragat 1, 44122 Ferrara, Italy. Tel.: +39.0532.974223.

E-mail: ddmgnn@unife.it

Key words: Cerebral venous drainage; internal jugular vein; central venous pressure; jugular vein pulse.

Acknowledgments: we acknowledge the doctoral program of the University of Ferrara for giving Nadiya Mohammed an opportunity to accomplish her doctoral thesis. This work is part of her PhD research in Department of Physics and Earth Science, University of Ferrara. Moreover, we thank our lab group members: Adriano Contillo, Paolo Cardarelli, and Giacomo Gadda for their invaluable contribution.

Contributions: NYM had framed the writing under supervision of MG and GDD.

Conflict of interests: the authors declare no conflict of interests.

Received for publication: 6 July 2019

Revision received: 2 September 2019.

Accepted for publication: 12 September 2019.

This work is licensed under a Creative Commons Attribution 4.0 License (by-nc 4.0).

CCopyright: the Author(s), 2019

Licensee PAGEPress, Italy

Veins and Lymphatics 2019; 8:8379

doi:10.4081/vl.2019.8379

multisepta, flaps, and malformed venous valves such as long, ectopic, accessory or fused leaflet, inverted valves, and double valves. ${ }^{24,26}$

\section{Neck vein cerebral outflow}

The main cerebral venous outflow of the neck consists of the IJVs and vertebral venous system, and the deep cervical veins (cervical soft tissue veins). Their pathways show multiple anastomoses between them in the neck, especially in the region of the cranial cervical junction. IJVs are the largest veins in the neck and considered the most important cerebral blood collectors in supine position. ${ }^{27-29}$

The external jugular vein (EJV) and anterior jugular vein (AJV), compared with the IJV, are located superficially in the neck. EJV is formed by the confluence of the posterior branch of the posterior facial vein and the posterior auricular vein. It usually terminates into the confluence of the subclavian 
and IJV. ${ }^{30}$ The AJV receives blood from superficial veins, such as EJVs, facial veins or IJVs. They usually end in the subclavian vein or EJV. Bilateral AJVs may communicate via the jugular venous arch (JVA), which is located just above the sternum. The JVA receives tributaries from the thyroid gland via inferior thyroid veins. ${ }^{26,31}$

EJV and AJV serve as collaterals and become prominent when the main cerebral venous drainage pathways (IJV and vertebral veins VV) are compromised. ${ }^{32}$ The types and prevalence of human cerebral venous outflow patterns are analyzed by ultrasound US and magnetic resonance imaging MRI. . $8,33,34^{2}$

\section{Internal jugular vein morphology}

Internal jugular vein is the main vein collecting blood from the head and neck area. ${ }^{35,36}$ The cerebral venous flow goes mainly from the superficial and deep venous system to the transverse sinus that in its turn continues in the sigmoid sinus to drain at the level of IJV foramen, the initial start of IJV. The IJV is asymmetric with two slight bulbs at the terminations, the inferior bulb and superior bulb. It leaves the skull at jugular foramen to descend down the neck, beings at one end lateral to the interior carotid artery and then lateral to the common carotid artery. ${ }^{37}$ At the base of the neck it joins the subclavian vein to form the brachiocephalic vein (Figure 1).

The confluence of the two brachiocephalic veins gives rise to the superior vena cava, which drains the cerebral venous blood into the right atrium. ${ }^{38}$

\section{Jugular vein pulse pulse wave}

The jugular vein pulse (JVP) is the oscillating top of the vertical blood column in the distended proximal portion of IJV that reflects phasic pressure changes in vena cava or right atrial pressure, which is equivalent to right ventricle end diastolic pressure in the absence of tricuspid stenosis. ${ }^{9,39,40}$ The JVP provides valuable information about cardiac hemodynamics and filling pressure, characteristic wave patterns pathognomonic of cardiac diseases and indirect estimate of CVP. ${ }^{63}$

The right IJV is preferred to the left one in evaluating the JVP because firstly, the right IJV has a straight course line from superior vena cava to right atrium and secondly, it is less likely to be compressed from surrounding structures. Unlike the left IJV, it drains into innominate vein, which is not in straight line from vena cava and right atrium, and it might be compressed by dilated aorta or aneurysm. The normal mean jugular venous pressure is equivalent 6 to 8 $\mathrm{cm} \mathrm{H}_{2} \mathrm{O}$ that is determined as the height of blood column above the midpoint of the right atrium. ${ }^{40}$
The normal jugular venous pulse wave consists of three positive waves, $a, c$, and $v$, and two negative waves, $x$ and $y .{ }^{41,42}$

The (a) wave is caused by right atrial systole, when the pressure transmitted back to the jugular veins by the contraction of the right atrium followed by $(\mathrm{x})$ descent, which

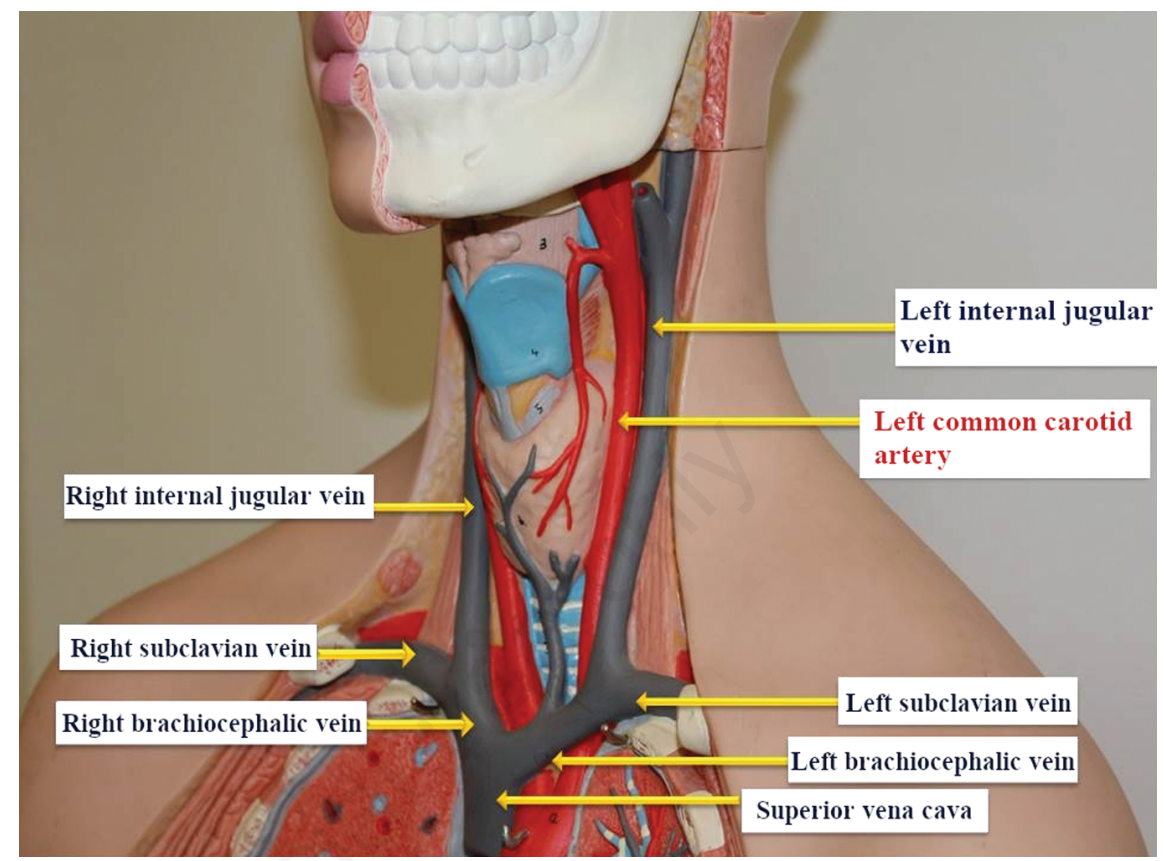

Figure 1. Anatomy of neck vessels including right and left jugular veins with their termination at brachiocephalic vein and vena cava. ${ }^{61}$

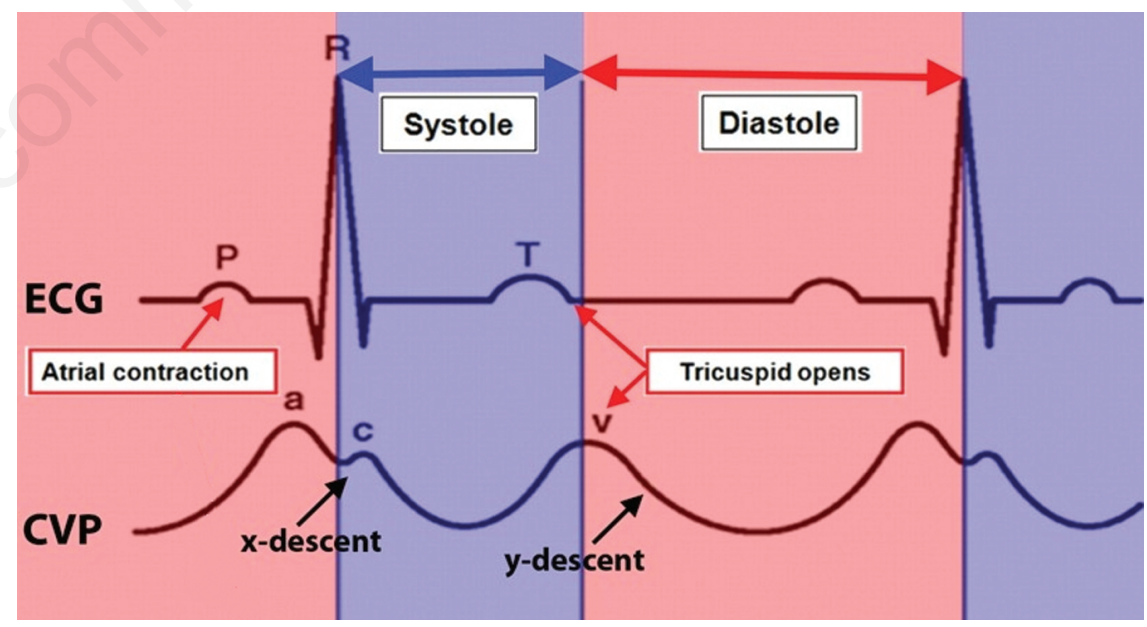

Figure 2. Normal JVP waveform corresponding to electrocardiography tracing ECG.The CVP tracings show three positive waves ( $a, c$ and $v$ ) and two descents wave ( $x$ and $y)$. (a wave) is due to the increased atrial pressure during right atrial contraction and it is correlated with the $P$ wave on ECG. (c wave) is caused by slight elevation of tricuspid valve into the right atrium during early ventricular contraction and it is correlated with the end of QRS segment in ECG. ( $x$ descent) is caused by downward movement of the ventricle during systolic contraction, it occurs before the $T$ wave in ECG. ( $v$ wave) arises from the pressure produced when the blood filling the right atrium come up against a closed tricuspid valve and it occurs at the end of $T$ wave in ECG. ( $y$ descent) is produced by the tricuspid valve opening with blood flowing into the right ventricle and it occurs before the $P$ wave in ECG. Reproduced with permission from: McNaugthon and Abu-Yousef, 2011. ${ }^{12}$ 
results from right atrial relaxation during atrial diastole and right ventricle systole. The (c) wave occurs in (x) descent due to the carotid artefact and Tricuspid valve pushing back caused by ventricular contraction. The (v) wave reflects the passive increase in pressure and volume of the right atrium as it fills in late systole when the tricuspid valve is still closed. ${ }^{41}$ It starts late during right ventricular systole and ends at early diastole. ${ }^{9,43}$ The (y) descent represents the abrupt termination of the down stroke of the (v) wave during early diastole after the tricuspid valve reopens and the right ventricle begins to fill passively. Normally the (y) descent is neither as brisk nor as deep as the $\mathrm{x}$ descent $^{42}$ (Figure 2).

\section{Ultrasound evaluation of internal jugular vein}

The internal jugular veins (IJVs), together with the vertebral veins constitute the predominant extra cranial pathways for the cerebral venous drainage. ${ }^{44,45} \mathrm{IJV}$ is moderately superficial, thus high frequency ultrasound linear probe is appropriate to obtain an anatomical image. B-mode can display the IJV along long axis view or short axis view, a perpendicular visualization of the vein, showing the IJV and carotid artery as rounded structures. ${ }^{45-47}$

The IJV size and course are highly variable, and it is easily compressed due to its thin wall and low blood pressure. ${ }^{47,48}$ Therefore, cross-sectional area (CSA) with subject head rotation, breathing, cardiac function and posture position.

As in supine, the CSA is largest, characterized by a positive value of hydrostatic pressure. In upright, the pressure becomes negative, and the external component of the transmural pressure, presented by the atmospheric pressure, becomes prevalent and press the IJV wall. This increased external pressure over the IJV results in a significantly reduced CSA. ${ }^{49}$

Consequently, the color Doppler ultrasonography generally used for IJV screening, is operator dependent and limited in its field of view..$^{50,52,53}$ A normal jugular vein gradually reduces its longitudinal diameter, as described in anatomy books, ${ }^{8}$ it is possible to segment IJV into three different segments $J_{3}$ to $J_{1}$, as it proposed in ultrasound US studies and CT $\operatorname{scan}^{3,54,55}$ (Figure 3). Anatomically speaking, left and right lower jugular segments (J1) correspond to the segments close to the junction of the internal jugular veins with the subclavian vein, at the confluence with the brachiocephalic vein trunk. The middle segments (J2) corre-

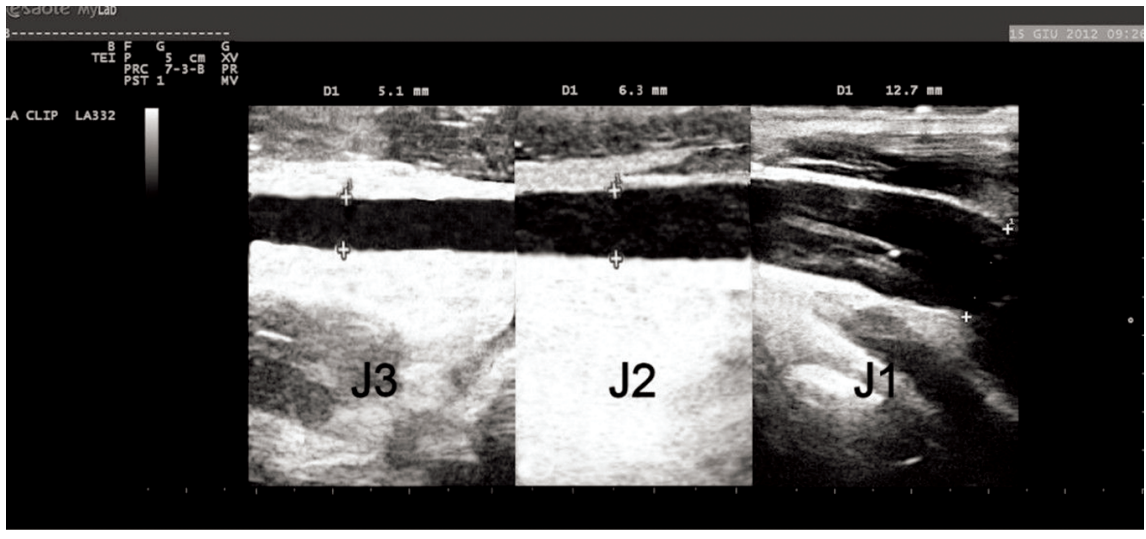

Figure 3. Normal internal jugular vein, long axis view showing progressive reduction of vessel diameter proceeding from $\mathrm{J} 1$ point $(12.7 \mathrm{~mm})$ passing through $\mathrm{J} 2(6.3 \mathrm{~mm})$ to $\mathrm{J} 3$ $(5.1 \mathrm{~mm}) .^{3}$

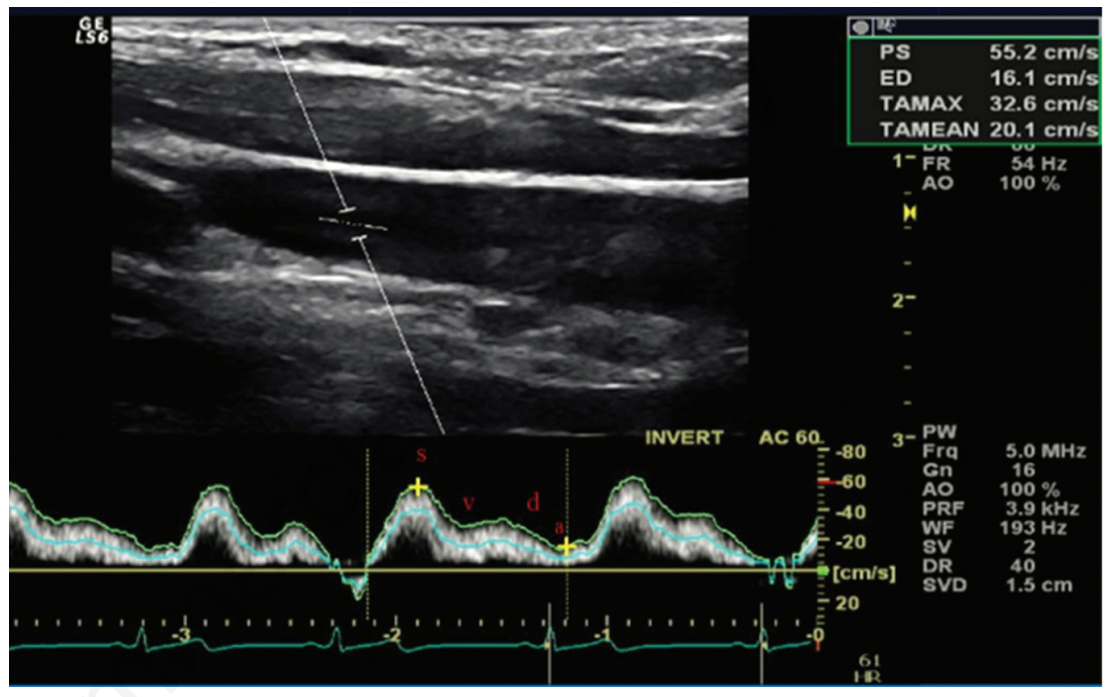

Figure 4. Doppler spectrum waves of internal jugular vein in section J3. Systolic wave (s), depressed velocity at mid diastole (v), late diastole wave (d) and end diastolic slowing wave (a).

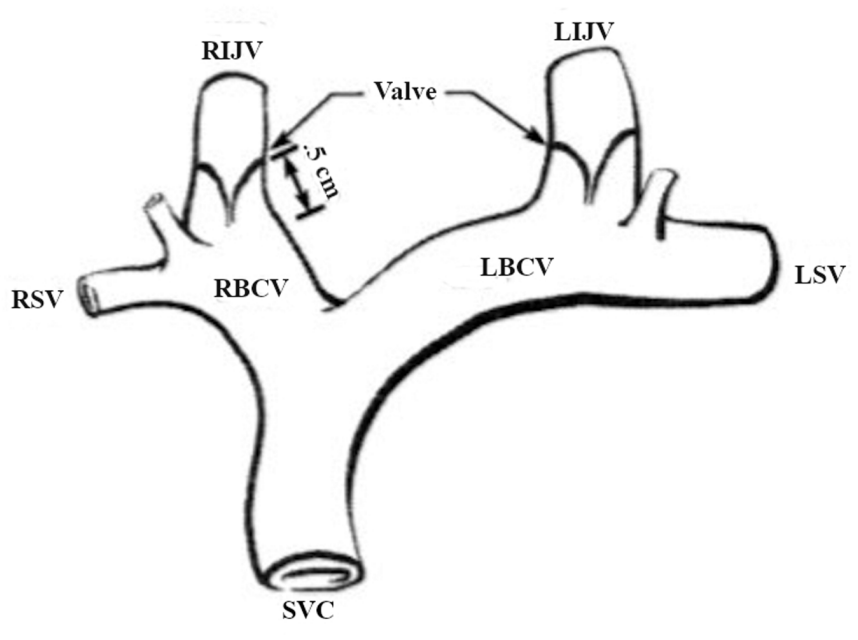

Figure 5. Anatomical diagram of internal jugular valves. Right internal jugular vein (RIJV), left internal jugular vein (LIJV); right subclavian vein (RSV); left subclavian vein (LSV), superior vena cava (SVC), right brachiocephalic vein (RBCV); left brachialcephalic vein (LBCV). Reproduced with permission from: Ratanakorn et al., 1999.62 
spond to the point where the veins are in an anatomical relationship with the more lateral contour of the thyroid gland. The upper segments (J3) correspond to the point before the passage through the jugular foramen into the skull. 8,52

\section{Doppler blood velocity of inter- nal jugular vein}

The ultrasound examination began with transverse scans to identify the jugular veins and carotid arteries. Subsequently, the IJVs were scanned in the longitudinal view to assess the magnitude and morphology of intraluminal echoes. Cardiac contractions and intrathoracic pressure changes are reflected in Doppler spectrum of the internal jugular vein. During expiration or the Valsalva maneuver, intrathoracic pressure increases, leading to reduced venous return and an increased diameter of the internal jugular vein, little or no flow is seen at such times. During inspiration, the venous flow is increased as a result of negative intrathoracic pressure and produces a higher-amplitude spectrum. ${ }^{55,56}$

The venous Doppler spectrum consists of (S), (v), (D), and (a) waves that are normally discernible (Figure 4).

$(S)$ wave is the systolic wave resulting from negative intra-atrial pressure with movement of the atrioventricular septum toward the cardiac apex. $(v)$ wave is caused by positive intra-atrial pressure produced by overfilling of the right atrium. $(D)$ is the diastolic wave, which determines by negative intra-atrial pressure, consequence of the opening of the tricuspid valve. (a) wave reflects positive intra-atrial pressure during atrial contraction. ${ }^{57-59}$

\section{Internal jugular vein valve mor- phology}

The valve cusps are macroscopically described in anatomical studies as thin translucent structures; the majority of valves are composed of two cusps (66-90\%).

Mono cusp valves are the second most common form, while tricuspid valves account for $6-7 \%{ }^{60}$ The valves are located on the distal portion of the IJV (J1, in the ultrasound) $0.5 \mathrm{~cm}$ above the union of the subclavian and internal jugular veins at the lower limit of the jugular bulb as it presents in Figure 5..$^{58,59}$ Using ultrasound imaging, the IJV valve were found in one IJV in about $90 \%$ of the cases, more frequently on the right side. ${ }^{60-62}$

\section{References}

1. Valdueza JM, von Münster T, Hoffman $\mathrm{O}$, et al. Postural dependency of the cerebral venous outflow. Lancet 2000;355:200-1.

2. Barami K. Cerebral venous overdrainage: an under-recognized complication of cerebrospinal fluid diversion. Neurosurg Focus 2016; 41:E9.

3. Farina M, Novelli E, Pagani R. Crosssectional area variations of internal jugular veins during supine head rotation in multiple sclerosis patients with chronic cerebrospinal venous insufficiency: a prospective diagnostic controlled study with duplex ultrasound investigation. BMC Neurol 2013;13: 162-74.

4. Vailati D, Lamperti M, Subert M, Sommariva A. An ultrasound study of cerebral venous drainage after internal jugular vein catheterization. Crit Care Res Pract 2012;2012:1-5.

5. Piechnik SK, Czosnyka M, Richards HK, et al. Cerebral venous blood outflow: a theoretical model based on laboratory simulation. Neurosurgery 2001; 49:1214-23.

6. Zamboni P, Consorti G, Galeotti R, et al. Venous collateral circulation of the extracranial cerebrospinal outflow routes. Curr Neurovasc Res 2009:20412.

7. Zamboni P, Menegatti E, Galeotti R, et al. Chronic cerebrospinal venous insufficiency. Ital J Vasc Endovasc Surg 2018;25:190-8.

8. Zamboni P, Morovic S, Menegatti E, et al. Screening for chronic cerebrospinal venous insufficiency (CCSVI) using ultrasound--recommendations for a protocol. Int Angiol 2011;30:571-97.

9. Garg N, Garg N. Jugular venous pulse : an appraisal. Indian Acad Clin Med 2000;1:260-9.

10. Weyland A, Grüne F. Cardiac preload and central venous pressure. Anaesthesist 2009;58:506-12.

11. Chua Chiaco JMS, Parikh NI, Fergusson DJ. The jugular venous pressure revisited. Cleve Clin J Med 2013;80:638-44.

12. McNaugthon DA, Abu-Yousef MM. Doppler US of the liver made simple. RadioGraphics 2011;31:161-88.

13. Sisini F. Physical description of the blood flow from the internal jugular vein to the right atrium of the heart: new ultrasound application perspectives. arXiv 2016;1604.05171.

14. Sisini F, Tessari M, Menegatti E, et al.
Clinical applicability of assessment of jugular flow over the individual cardiac cycle compared with current ultrasound methodology. Ultrasound Med Biol 2016;42:1750-63.

15. Guarracino F, Ferro B, Forfori F, et al. Jugular vein distensibility predicts fluid responsiveness in septic patients. Crit Care 2014;18:647.

16. Broilo F, Meregalli A, Friedman G. Right internal jugular vein distensibility appears to be a surrogate marker for inferior vena cava vein distensibility for evaluating fluid responsiveness. Rev Bras Ter Intensiva 2015;27:205-11.

17. Holmlund P, Johansson E, Qvarlander $\mathrm{S}$, et al. Human jugular vein collapse in the upright posture: implications for postural intracranial pressure regulation. Fluids Barriers CNS 2017;14:1723.

18. Sisini F, Toro E, Gambaccini M, Zamboni $\mathrm{P}$. The oscillating component of the internal jugular vein flow: the overlooked element of cerebral circulation. Behav Neurol 2015;2015:1-9.

19. Zamboni P. Why current doppler ultrasound methodology is inaccurate in assessing cerebral venous return: the alternative of the ultrasonic jugular venous pulse. Behav Neurol 2016;2016:1-7.

20. Lin SK, Chang YJ, Yang FY. Hemodynamics of the internal jugular vein: an ultrasonographic study. Tzu Chi Med J 2009;21:317-22.

21. Menegatti E, Tessari M, Gianesini S, et al. Human internal jugular valve Mmode ultrasound characterization. Curr Neurovasc Res 2014;11:149-55.

22. Stolz E. Ultrasound examination techniques of extra- and intracranial veins. Perspect Med 2012;1:366-70.

23. Hsu HY. Jugular venous reflux and neurological disorders. Acta Neurol Taiwan 2011;20:1-3.

24. Zhou D, Ding J, Ya J, et al. Understanding jugular venous outflow disturbance. CNS Neurosci Ther 2018;24(6):473-82.

25. Doepp F, Valdueza J, Schreiber S. Incompetence of internal jugular valve in patients with primary exertional headache: a risk factor? Cephalalgia 2008;28:182-5.

26. Zivadinov R, Chung CP. Potential involvement of the extracranial venous system in central nervous system disorders and aging. BMC Med 2013;11:260.

27. Toro E, Muller L, Cristini M, et al. Impact of jugular vein valve function on cerebral venous haemodynamics. Curr Neurovasc Res 2015;12:384-97. 
28. Doepp F, Schreiber SJ, von Münster T, et al. How does the blood leave the brain? A systematic ultrasound analysis of cerebral venous drainage patterns. Neuroradiology 2004;46:565-70.

29. Zamboni P, Menegatti E, Pomidori L, et al. Does thoracic pump influence the cerebral venous return? J Appl Physiol 2012;112;904-10.

30. Deslaugiers B, Vaysse P, Combes JM, et al. Contribution to the study of the tributaries and the termination of the external jugular vein. Surg Radiol Anat 1994;16:173-7.

31. Schummer W, Schummer C, Bredle D, et al. The anterior jugular venous system: variability and clinical impact. Anesth Analg 2004;99:1625-9.

32. Escott EJ, Branstetter BF. It's not a cervical lymph node, it's a vein: $\mathrm{CT}$ and MR imaging findings in the veins of the head and neck. Radiographics 2006;26:1501-15.

33. Nicolaides AN, Morovic S, Menegatti $\mathrm{E}$, et al. Screening for chronic cerebrospinal venous insufficiency (CCSVI) using ultrasound: recommendations for a protocol. Funct Neurol 2011;26:229-48.

34. Simka M, Ludyga T, Latacz P, et al. Diagnostic accuracy of current sonographic criteria for the detection of outflow abnormalities in the internal jugular veins. Phlebol J Venous Dis 2013;28:285-92.

35. Deepak CA, Sarvadnya JJ, Sabitha KS. Variant anatomy of internal jugular vein branching. Ann Maxillofac Surg 2015;5:284-6.

36. Rivard AB, Burns B. Anatomy, head and neck, internal jugular vein. StatPearls Publishing. Available from: https://www.ncbi.nlm.nih.gov/books/N BK513258/

37. Oliver J. Venous drainage of the head and neck - Dural Sinuses TeachMeAnatomy. Available from http://teachmeanatomy.info/neck/vessels/venous-drainage/28

38. Gray. The veins of the neck - human anatomy. 2015. Available from: https://theodora.com/anatomy/the_vein S of the neck.html

39. Karnath B, Thornton W, Beach R. Inspection of neck veins. Hosp Physician 2002;May:43-7.

40. Izakovic M. Central venous pressure evaluation, interpretation, monitoring, clinical implications. Bratisl Lek List 2008;109:185-7.

41. Devine PJ, Sullenberger LE, Bellin DA, Atwood JE. Jugular venous pulse: window into the right heart. South Med J 2007;100:1022-7.

42. Applefeld MM. The jugular venous pressure and pulse contour. clinical methods: the history, physical, and laboratory examinations. Boston: Butterworths; 1990.

43. Sisson C, Nagdev A. Focus on: ultrasound-guided central venous access of the internal jugular vein. ACEP News 2007; November:12-14.

44. Mahan AF, McEvoy MD, Gravenstein $\mathrm{N}$. Long-axis view for ultrasound-guided central venous catheter placement via the internal jugular vein. Rom J Anaesth Intensive Care 2016;23:27-31.

45. Miki I, Murata S, Nakazawa K, et al. Anatomical relationship between the common carotid artery and the internal jugular vein during head rotation. Ultrasound 2014;22:99-103.

46. Laganà MM, Pelizzari L, Scaccianoce E, et al. Assessment of internal jugular vein size in healthy subjects with magnetic resonance and semiautomatic processing. Behav Neurol 2016;2016:1-7.

47. Tain RW, Ertl-Wagner B, Alperin N. Influence of the compliance of the neck arteries and veins on the measurement of intracranial volume change by phasecontrast MRI. J Magn Reson Imaging 2009;30:878-83.

48. Zavoreo I, Bašić-Kes V, ZadroMatovina L, et al. Cerebral venous circulatory system evaluation by ultrasonography. Acta Clin Croat 2013;52:203-11.

49. Zamboni P, Tavoni V, Sisini F, et al. Venous compliance and clinical implications. Veins Lymphat 2018;7:49-55.

50. Lorchirachoonkul T, Ti LK, Manohara $\mathrm{S}$, et al. Anatomical variations of the internal jugular vein: Implications for successful cannulation and risk of carotid artery puncture. Singapore Med J 2012;53:325-8

51. Gadda G, Taibi A, Sisini F, et al. A new hemodynamic model for the study of cerebral venous outflow. Am J Physiol Heart Circ Physiol 2015;308:217-31.

52. Malferrari G, Prati P, Zedde M. Neurosonological evaluation of cere- bral venous outflow. Milan: SpringerVerlag; 2014.

53. Sivaciyan V, Ranganathan N. Transcutaneous Doppler jugular venous flow velocity. Circulation 1978;57:9309.

54. Valdueza J. Assessment of the venous system by ultrasound. In: Hands-on Course 5 Ultrasound in Vascular Diagnosis Level 1. 2018. Available from: https://www.ean.org /lisbon2018/fileadmin/user_upload/Ho C05_03_Valdueza.pdf

55. Schaller B. Physiology of cerebral venous blood flow: from experimental data in animals to normal function in humans. Brain Res Rev 2004;46:24360.

56. Chung $\mathrm{CP}, \mathrm{Hu} \mathrm{HH}$. Jugular venous reflux. J Med Ultrasound 2008;16:21022.

57. Chavhan GB, Parra DA, Mann A, et al. Normal Doppler spectral waveforms of major pediatric vessels: specific patterns. RadioGraphics 2008;28:691-706.

58. Dalla Vestra M, Grolla E, Bonanni L, et al. From the veins to the heart: a rare cause of varicose veins. Case Rep Vasc Med 2015;2015:1-3.

59. Kalmanson D, Veyrat C, Derai C, et al. Non-invasive technique for diagnosing atrial septal defect and assessing shunt volume using directional Doppler ultrasound. Correlations with phasic flow velocity patterns of the shunt. Br Heart J 1972;34:981-91.

60. Brownlow RL, McKinney WM. Ultrasonic evaluation of jugular venous valve competence. J Ultrasound Med 1985;4:169-72.

61. Donia A. Introduction to insertion of temporary IJ line; 2017. Available from: https:/www.slideshare.net/Melkholy/in terventional-nephrology-dr-ahmeddonia

62. Ratanakorn D, Tesh PE, Tegeler CH. A new dynamic method for detection of internal jugular valve incompetence using air contrast ultrasonography. J Neuroimaging 1999;9:10-14.

63. Sisini F, Tessari M, Gadda G, et al. An ultrasonographic technique to assess the jugular venous pulse: a proof of concept. Ultrasound Med Biol 2015;41: 1334-41. 